\title{
Multimodal Imaging for the Assessment of Geographic Atrophy in Patients with "Foveal" and "No-Foveal" Sparing
}

\author{
Stamatina A. Kabanarou ${ }^{a}$ Georgios Bontzos $^{a}$ Tina Xirou $^{a} \quad$ Zoi Kapsala ${ }^{b}$ \\ Eleni Dimitriou ${ }^{c}$ Panagiotis Theodossiadis ${ }^{c}$ Irini Chatziralli ${ }^{c}$ \\ aDepartment of Ophthalmology, Korgialenio Benakio Hospital, Athens, Greece; ${ }^{b}$ Department of Ophthalmology, \\ Hippokration General Hospital, Athens, Greece; ' 2 nd Department of Ophthalmology, National and Kapodistrian \\ University of Athens, Athens, Greece
}

\section{Keywords}

Geographic atrophy $\cdot$ OCTA $\cdot$ En-face OCT $\cdot$ Multimodal imaging · Foveal sparing

\begin{abstract}
Introduction: The aim of the study was to evaluate the applicability of optical coherence tomography (OCT) angiography (OCTA) for measuring geographic atrophy (GA) areas in age-related macular degeneration (AMD) patients with "foveal" and "no-foveal" sparing disease and compare it to other imaging modalities. Methods: A multimodal imaging protocol was applied, using infrared (IR) imaging, fundus autofluorescence (FAF), OCTA, and en-face OCT in 35 eyes of 23 AMD patients with GA. Patients were classified into 2 groups, with and without foveal sparing disease. GA area measurements for all imaging modalities were compared for each group separately. Results: The measured GA area was estimated to be $6.68 \pm 3.18 \mathrm{~mm}^{2}$ using $\mathrm{IR} ; 6.99 \pm 3.09 \mathrm{~mm}^{2}$ using FAF; $6.56 \pm 3.11 \mathrm{~mm}^{2}$ using OCTA, and $6.65 \pm 3.14 \mathrm{~mm}^{2}$ using en-face OCT. There was no statistically significant difference in the GA area between different modalities ( $p=0.977$ ). When separate analysis was conducted for patients with "foveal" and "no-foveal" sparing disease, although GA measure-
\end{abstract}

karger@karger.com

(c) 2020 S. Karger AG, Basel

www.karger.com/ore

Karger" ments in FAF imaging displayed higher numerical values than the other modalities, especially in patients with foveal sparing, no statistically significant difference in the GA area was found between the different imaging modalities in either group ( $p=0.816$ for foveal sparing; $p=0.992$ for no-foveal sparing group). Conclusions: OCTA can be reliably used in the assessment of GA in AMD patients with and without foveal sparing disease. For both groups, measurements are comparable to IR, en-face OCT, and FAF, despite the fact that the latter recorded larger area of GA, mainly in the foveal sparing cases.

(c) 2020 S. Karger AG, Basel

\section{Introduction}

Age-related macular degeneration (AMD) is the leading cause of visual impairment and irreversible visual loss in the elderly population in developed countries [1]. Approximately $20 \%$ of AMD patients with severe visual acuity present with geographic atrophy (GA), the end-stage manifestation of nonexudative AMD, leading to central vision loss [2]. To date, no evidence-based proven therapy has been established for the treatment or prevention of GA $[3,4]$. 
GA is characterized by the progressive loss of outer retinal layers and the retinal pigment epithelium (RPE) [5], which can be also accompanied by loss of the underlying choriocapillaries during disease progression [6]. Specifically, GA has been defined as a round or oval atrophic area of $175 \mu \mathrm{m}$ or more in diameter [7]. This process may start as patches of GA at the macula or typically at the parafoveal area, which eventually expand to the fovea, while multiple atrophic areas may occur over time [8]. These areas tend to slowly enlarge and coalesce, often leaving the fovea uninvolved until a later stage of disease progression, referred as foveal sparing [6].

Previous and current imaging technologies of the posterior pole of the eye, such as color fundus photography, infrared (IR) reflectance imaging, optical coherence tomography (OCT), en-face OCT, fundus autofluorescence (FAF), and, more recently, OCT angiography (OCTA), have enabled in vivo visualization of retinal structures and anatomical evaluation of AMD pathology $[9,10]$. To observe and quantify in detail GA development and progression, it is critical to identify techniques for accurate and reliable calculations.

The gold standard technique for atrophy evaluation and progressive enlargement of GA is considered to be FAF [11]. Both manual and semiautomated methodologies have been proposed for measurements [12-14] and have been accepted as clinical tools to estimate disease pathology end points in multicenter clinical trials, such as Spectri and Chroma [4]. On FAF, areas of atrophy are distinguished from the healthy RPE due to the absence of fluorophores and are displayed as well-defined dark areas of reduced signal, allowing their quantification [15]. However, the normal fovea is also documented as a dark area due to reduced autofluorescence signal, especially prominent in blue-light autofluorescence. This effect is attributed to the presence of macular pigment carotenoids, lutein, zeaxanthin, and meso-zeaxanthin in the normal fovea, which absorb short-wavelength excitation light [16]. Therefore, evaluation of the fovea with FAF imaging in cases with GA at the macular area might be challenging.

An alternative approach to measure GA areas has been attempted using OCTA, which allows for detection of atrophic areas as loss of choriocapillary flow signal under the degenerated RPE and better visualization of the deeper choroidal vessels [17]. It has been shown that measurements are reliable and display high agreement with FAF, giving also the opportunity to exclude the coexistence of choroidal neovascularization [18].

Moreover, OCTA offers a more "accurate" measurement of the atrophic area, especially in cases with foveal sparing areas, as normal choriocapillary flow would be documented in the fovea in such cases. On the contrary, in FAF imaging, the area of the normally reduced autofluorescence signal in the fovea would be added to the dark signal of surrounded GA areas, contributing to false enlargement of the pathologic measured areas. In light of the above, the purpose of the present study is to evaluate the accuracy of OCTA measurements in GA quantification and to compare the results with gold standard FAF, as well as with IR and en-face OCT, separating patients with GA into 2 groups, based on the presence or absence of foveal sparing.

\section{Methods}

For this cross-sectional study, we examined 29 patients (43 eyes) with GA secondary to nonexudative AMD, who attended the Department of Ophthalmology of Korgialenio Benakio Hospital, Athens, and the Department of Ophthalmology, University of Athens, between March 2018 and August 2019. The study was in accordance with the tenets of Helsinki Declaration. Informed consent was obtained from all patients before enrollment to the study.

GA was initially diagnosed and defined by fundus examination as one or more well-defined areas of patchy atrophy of the RPE, typically with exposure of the underlying choroidal vessels [19]. Disease duration was reported from patients' charts since GA establishment. The exclusion criteria based on clinical examination and fundoscopy were (1) signs of choroidal neovascularization, with macular hemorrhage, intraretinal or subretinal fluid, and subretinal fibrosis; (2) any previous treatment including intravitreal injections or photodynamic therapy; (3) presence of any concomitant ocular pathology, like diabetic retinopathy, retinal vein or artery occlusion, and intraocular inflammation; and (4) high myopia (over 7D). Additional exclusion criteria based on imaging were GA area extended outside the central scanning area of $6 \times 6$ $\mathrm{mm}$ and the presence of significant media opacities that could potentially compromise OCTA or FAF image quality (e.g., corneal opacities or significant cataract).

After comprehensive ophthalmic examination, including Snellen best-corrected distance visual acuity (BCVA) and fundus biomicroscopy with a noncontact lens, the examined eyes were defined as having foveal sparing if the residual foveal island was $>270^{\circ}$ surrounded by well-demarcated areas of GA [20]. Following this documentation, all patients were divided into 2 groups: with and without foveal sparing. Subsequently, all patients underwent posterior pole IR, FAF, OCTA, and en-face OCT imaging.

\section{Imaging Protocol}

IR and FAF images were acquired with the Spectralis HRA + OCT (Heidelberg Engineering, Heidelberg, Germany) after a 20-S bleach of the photopigments in AF mode. Heidelberg utilizes confocal microscopy with blue light to obtain FAF images.

OCTA was performed using a commercially available RTVue XR Avanti with an AngioVue (Optovue, Fremont, CA, USA) with $6 \times 6-\mathrm{mm}$ images of the macula. For a more efficient visualization and measurement of the atrophic area delineated by the loss of 
choriocapillary flow signal under the degenerated RPE and visualization of the deeper choroidal vessels, a customized slab was selected. The upper boundary was defined at the RPE level and the lower boundary at $90 \mu \mathrm{m}$ below the RPE, slightly below the choriocapillaries for optimal visualization of this zone [21]. En-face OCT angiograms were selected at the same area.

Images were exported into ImageJ software version 1.50 (Rasband, W.S., US National Institutes of Health, Bethesda, MD, USA) for pixel-based measurements. Objective quantification was performed by 2 investigators (G.B. and Z.K.), using the following technique: the atrophic areas in digital images (Fig. 1a) were segmented by color thresholding based on values of color saturation, hue, and color intensity. In FAF, sharply demarcated areas of reduced autofluorescence were considered the atrophic area. In IR and en-face OCT, sharply demarcated areas of altered pigmentation were defined as the atrophic area [10]. In OCTA, the areas lacking choriocapillaries with improved visualization of the outer choroidal vasculature were considered the atrophic area [18]. The region of interest containing GA was manually marked and selected for measurement. The pixel area was then converted into square millimeters $\left(\mathrm{mm}^{2}\right)$ according to the scale of the image. For qualitative assessment of the marked areas, images were overlapped using anatomical landmarks of the posterior pole, especially the temporal arcades.

\section{Statistical Analysis}

Statistical analysis was performed using SPSS (IBM SPSS Statistics for Windows, version 22.0; Armonk, NY, USA). Descriptive statistics are presented as mean $\pm \mathrm{SD}$. Comparisons between modalities were performed with the Kruskal-Wallis $H$ test. Pairwise differences were examined by the Mann-Whitney $U$ test with a significance level of 0.05 .

To test the proportion of agreement among measurements, we tested intra-observer and inter-observer agreement as follows: intra-observer reliability of measurements was calculated by comparing 2 separate measurements performed by one observer 1 month apart to minimize recall bias. Inter-observer reliability of measurements was calculated by comparing area measurements of 2 separate observers, using the same methodology. The intraclass correlation coefficient (ICC) (2-way mixed model) was computed to estimate the inter-rater and intra-rater reliability [22]. The value of the ICC $>0.7$ in absolute single measures was considered to be an acceptable agreement.

In addition, the Bland-Altman statistical test was used to determine $95 \%$ limits of agreement between FAF and OCTA. The 95\% limits of agreement were defined as the mean difference $\pm 1.96 \mathrm{SD}$, where SD is the standard deviation of the differences. In all statistical analyses, $p$ values $<0.05$ were considered as statistically significant.

\section{Results}

This analysis included 35 eyes of 23 patients. Eight eyes of 6 patients failed to fulfill the imaging inclusion criteria and were excluded. The mean age of patients was $77.58 \pm$ 2.14 (range: 65-90) years, and all were Caucasians, and 10 patients were males and 13 females.

Multimodal Imaging in Geographic Atrophy

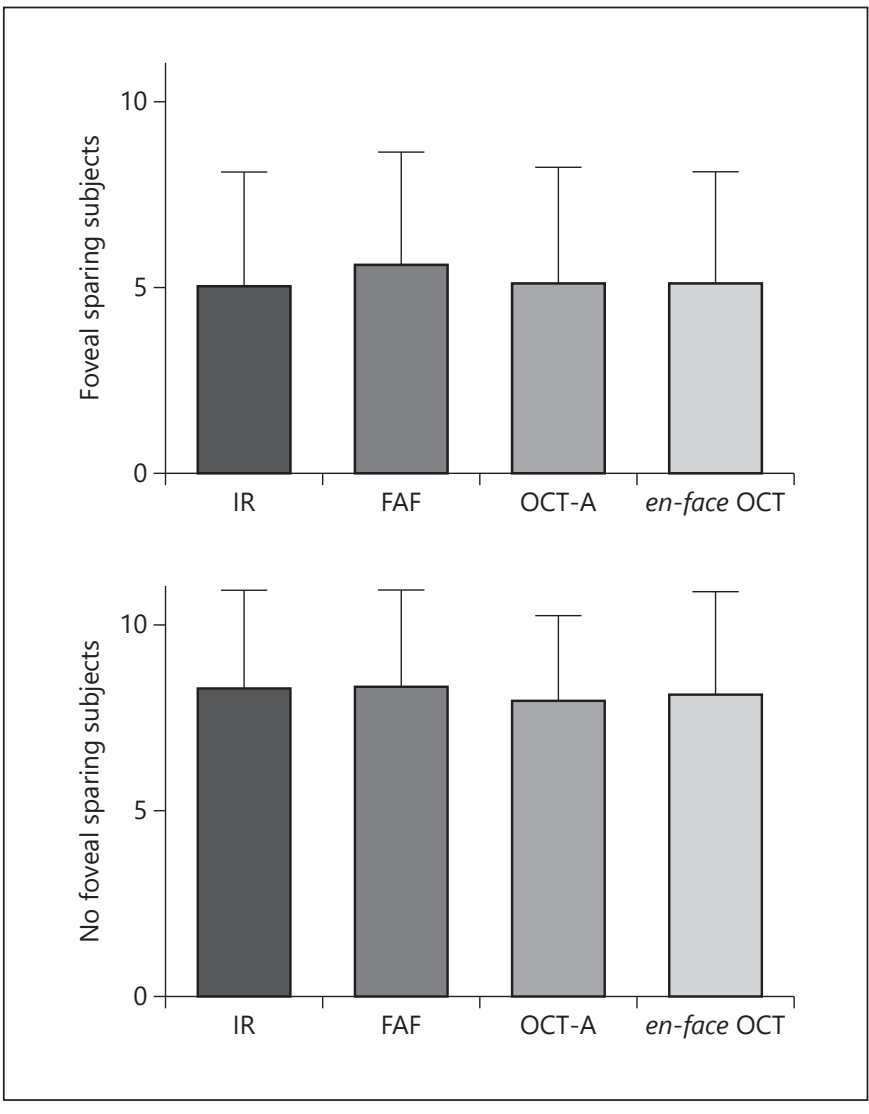

Fig. 1. Error bars showing mean $+\mathrm{SD}$ values of $\mathrm{GA}$ measurements for patients with foveal involvement or sparing using the different imaging modalities: IR, FAF, OCTA, and en-face OCT. OCTA, optical coherence tomography angiography; OCT, optical coherence tomography; IR, infrared; FAF, fundus autofluorescence.

The mean measured area of GA lesion was estimated to be $6.68 \pm 3.18$ (range: $0.97-16.88$ ) $\mathrm{mm}^{2}$ using IR, compared to $6.99 \pm 3.09$ (range: $1.02-16.39$ ) $\mathrm{mm}^{2}$ using FAF, $6.56 \pm 3.11$ (range: $0.87-16.41$ ) $\mathrm{mm}^{2}$ using OCTA, and $6.65 \pm 3.14$ (range: $0.97-16.75$ ) $\mathrm{mm}^{2}$ using en-face OCT. There was no statistically significant difference in the GA area between different modalities $\left(p=0.977, \chi^{2}=0.63\right.$; Kruskal-Wallis $H$ test). Post hoc analysis demonstrated no significant differences between each component of the comparisons $(p>0.05)$. The GA area as measured by OCTA was not significantly associated with age, gender, disease duration, and axial length $(p=0.674 ; p=0.672$; $p=0.241 ; p=0.455$, respectively). A weak, but significant, correlation was found between the GA area and BCVA $(r=0.311 ; p=0.03)$.

Furthermore, 16 eyes were classified as having "foveal" sparing and 19 eyes as "no-foveal" sparing. No statistically significant difference in the GA area between 

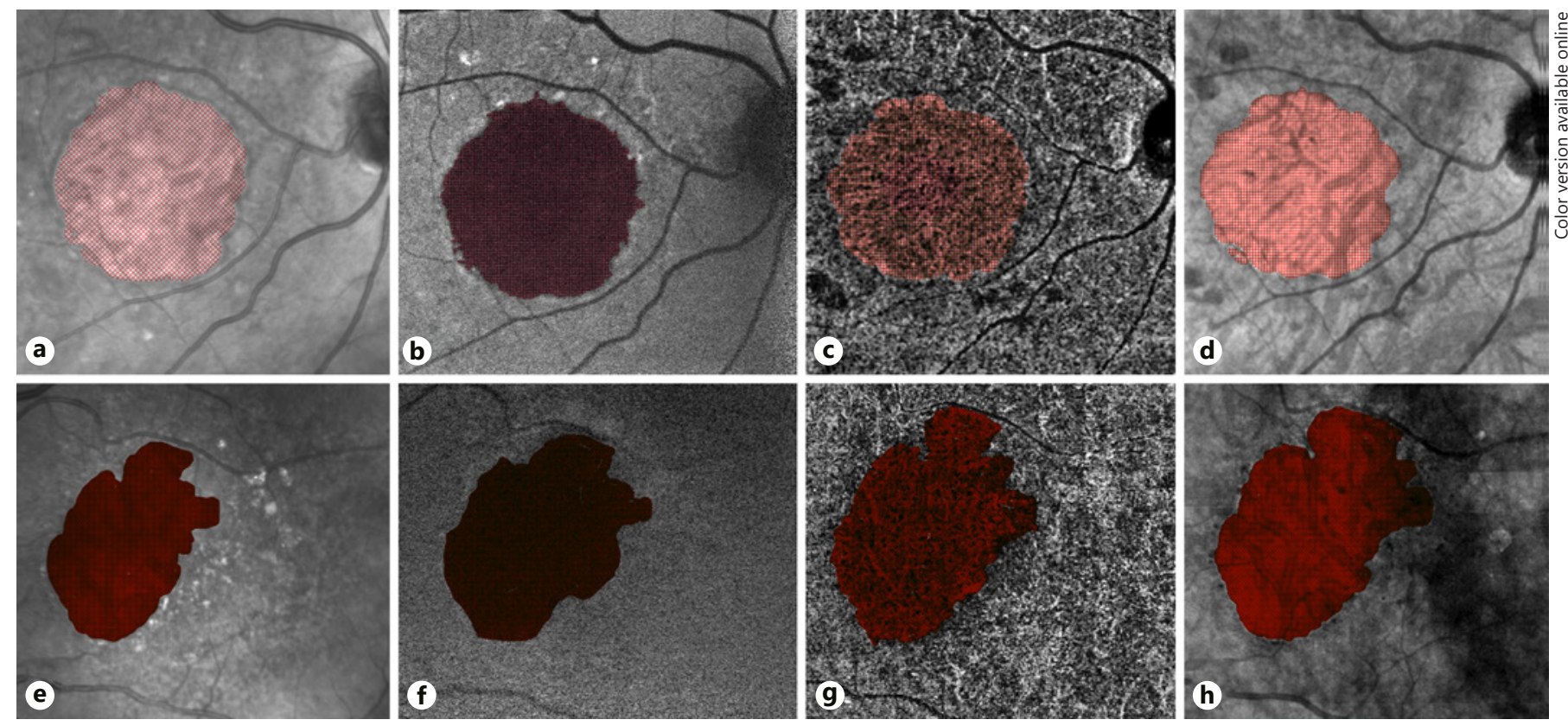

Fig. 2. Multimodal imaging in 2 patients with GA. In the upper panels, a case of "no-foveal" sparing GA imaged by IR (a), FAF (b), OCTA (c), and en-face OCT (d) is presented. In the lower panels, a patient with "foveal" sparing is shown. We can observe that in FAF, (f) a larger area spreading out temporally in the fovea area is demarcated as "atrophy" compared to IR (e), OCTA (g), and en-face OCT (h). OCTA, optical coherence tomography angiography; OCT, optical coherence tomography; IR, infrared; FAF, fundus autofluorescence.

Table 1. Mean GA area measurements as calculated by IR), FAF, OCTA, and en-face OCT are presented for the whole cohort, and for the foveal and no-foveal sparing groups separately

\begin{tabular}{llll}
\hline Modality & Whole cohort, $\mathrm{mm}^{2}$ & Foveal sparing, $\mathrm{mm}^{2}$ & No-foveal sparing, $\mathrm{mm}^{2}$ \\
\hline IR & $6.68 \pm 3.18$ & $5.01 \pm 3.11$ & $8.26 \pm 3.02$ \\
FAF & $6.99 \pm 3.09$ & $5.59 \pm 3.06$ & $8.31 \pm 2.69$ \\
OCTA & $6.56 \pm 3.11$ & $5.11 \pm 3.12$ & $7.93 \pm 2.68$ \\
En-face OCT & $6.65 \pm 3.14$ & $5.09 \pm 3.02$ & $8.11 \pm 2.79$ \\
\hline$p$ value* & 0.977 & 0.816 & 0.992 \\
\hline
\end{tabular}

OCTA, optical coherence tomography angiography; OCT, optical coherence tomography; IR, infrared; FAF, fundus autofluorescence. ${ }^{*}$ Kruskal-Wallis $H$ test. The $p$ values were presented for the comparisons among the different imaging modalities for each group.

different modalities was found in either group $(p=$ 0.816 for the "foveal" sparing group; $p=0.992$ for the "no-foveal" sparing group) (Fig. 1). The results of GA area measurements for all imaging modalities in the full cohort and in both the "foveal" sparing and "no-foveal" sparing groups are shown in Table 1. Figure 2 shows representative examples of the imaging techniques and measurements in patients with and without foveal sparing.

\section{Intra-Observer and Inter-Observer Agreement}

Regarding intra-observer measurements for foveal sparing disease, there was no statistically significant difference in the mean GA area between the first and the second evaluation for the first investigator as far as IR $(p=0.885), \mathrm{FAF}(p=0.924)$, OCTA $(p=0.728)$, and enface OCT ( $p=0.923)$ are concerned, while the ICC agreement was excellent (ICC $\geq 0.991 ; p<0.001$ for all comparisons). Accordingly, for no-foveal sparing disease, 
there was no statistically significant difference in the mean GA area measurement between the first and the second evaluation for the first investigator in IR ( $p=$ $0.811), \operatorname{FAF}(p=0.842)$, OCTA $(p=0.834)$, and en-face OCT $(p=0.862)$ with high ICC agreement too (ICC $\geq 0.99 ; p<0.001$ for all comparisons). For inter-observer agreement, we found high inter-rater agreement between the measurements of the second investigator and the first investigator for both foveal and no-foveal sparing disease (ICC $\geq 0.98 ; p \leq 0.01$ for all comparisons).

The exact limits of agreement between OCTA and the other imaging modalities are presented in the Bland-Altman plots in Figure 3. The mean difference in the GA area using IR and OCTA was $0.119 \pm 0.628 \mathrm{~mm}^{2}$, defining the limits of agreement from -1.111 to $1.35 \mathrm{~mm}^{2}$. The mean difference in the GA area using FAF and OCTA was 0.426 $\pm 0.674 \mathrm{~mm}^{2}$, defining the limits of agreement from -0.894 to $1.748 \mathrm{~mm}^{2}$. Finally, the mean difference in the GA area using en-face OCT and OCTA was $0.088 \pm 0.437$ $\mathrm{mm}^{2}$, with limits of agreement from -0.769 to $0.946 \mathrm{~mm}^{2}$.

\section{Discussion}

In this analysis, we included 35 eyes of 23 patients in order to assess the use of OCTA to detect and accurately quantify GA in AMD patients. We also compared its results with other imaging modalities, such as IR, FAF, and en-face OCT. Moreover, we examined the applicability of all the aforementioned imaging devices to measure the GA areas both in foveal sparing and no-foveal sparing cases. Our study showed that OCTA is an effective tool to estimate GA, and all 4 methods produced similar results, with no statistical difference among their measurements. However, the mean area of GA as measured by FAF was larger in the whole cohort than that measured by the other 3 devices, but this difference did not reach a statistically significant level. When mean areas were compared separately in the "foveal" and in the "no-foveal" sparing group, it was evident that the range of the difference found between FAF and the other imaging modalities in the whole cohort was mainly driven by the range of the difference noticed in the foveal sparing group (between FAF measurements and the other 3 techniques) as this difference was much smaller in the no-foveal sparing group. Therefore, FAF could potentially overestimate the GA area compared to other imaging modalities in such cases. This could probably be explained by the fact of the normal macula pigments, reducing the FAF signal [23], and this anatomically "normal" area is added to the dark

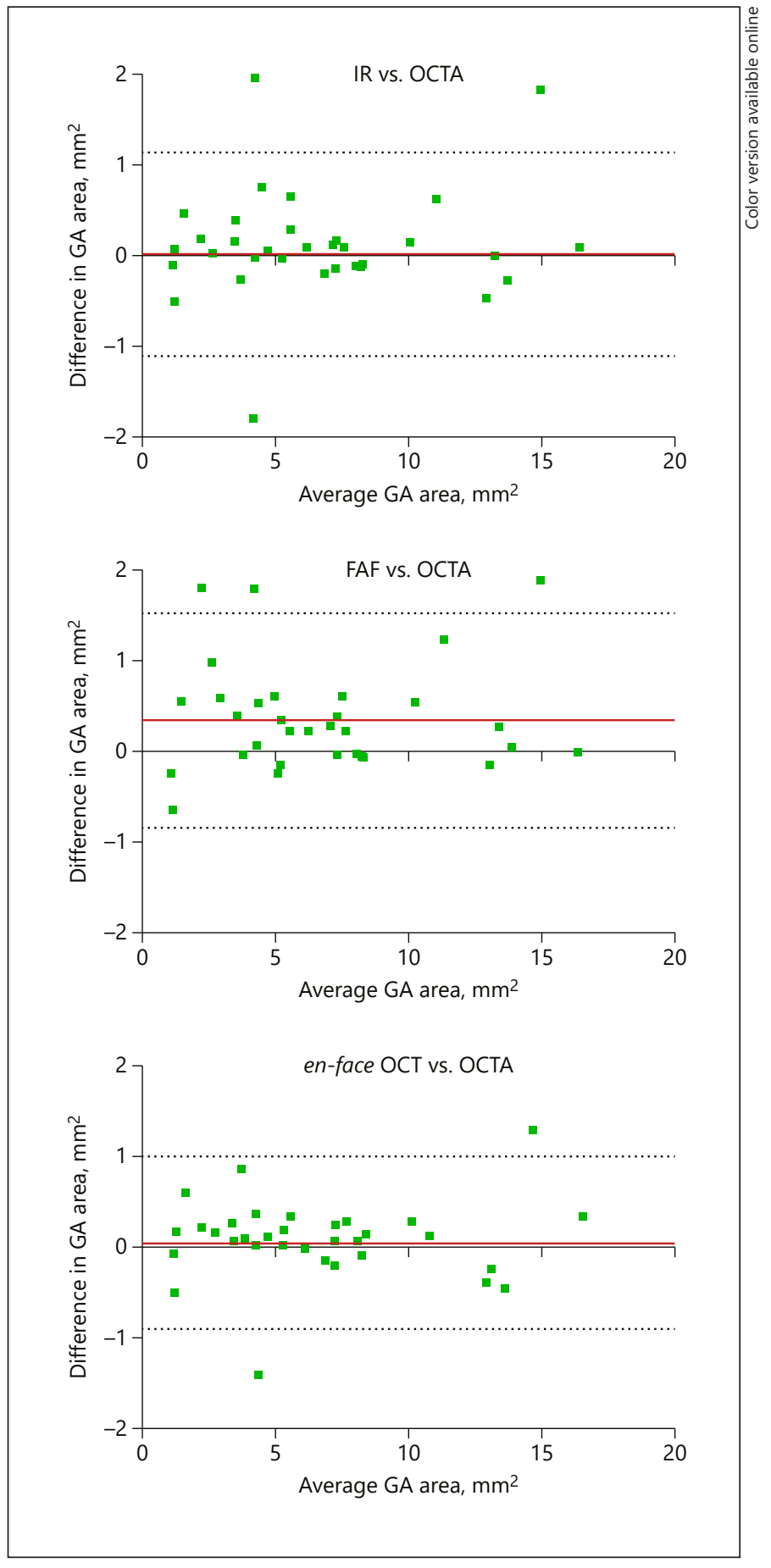

Fig. 3. Bland-Altman plots demonstrating the exact limits of agreement between OCTA and the other imaging modalities: Differences in GA area estimates as defined by IR and OCTA (upper plot), FAF and OCTA (mid plot), and en-face OCT and OCTA (lower plot) are presented against average GA area. The mean difference in GA is presented with the solid gray line, whereas the $95 \%$ limits of agreement are shown with the dotted lines. OCTA, optical coherence tomography angiography; OCT, optical coherence tomography; IR, infrared; FAF, fundus autofluorescence. 
signal of adjacent GA areas, causing an overestimation of the pathologic measured areas. Yet, this difference in mean areas was not statistically significant in either group. Likewise, all methods proved to be highly reproducible with intra-rater and inter-rater reliability in the whole cohort and in each group separately.

In the previous years, fundus photography was considered as the standard method for quantification and evaluation of disease progression in GA [24]. Large epidemiological studies were based on fundus photography to observe the natural history of the disease $[8,25]$. However, measurements were prone to subjective bias, as the GA margins were not always easy to delineate from the adjacent healthy retinal tissue [26], while the presence of drusen could lead to further measurement errors [27]. In addition, foveal sparing identification was not clear at that time [26], but when using multicolor imaging [28], as reported afterward, more accurate results are documented in such cases.

Advances in retinal imaging and the introduction of FAF provide high-contrast images of GA areas, allowing the accurate segmentation of the atrophic areas [29]. Nevertheless, low-FAF signal intensities in the foveal area because of short-wavelength light absorption make quantification challenging [23]. To address this issue, researchers have tried to combine images from FAF and SD-OCT [30] to better illustrate both the borders of the atrophy as well as the foveal involvement. Additionally, FAF imaging using different wavelength has been described [31]. A recent study by Borelli et al. [32] compared short-wavelength FAF with conventional FAF, showing promising results, especially in cases of foveal sparing disease.

An alternative approach to acquire GA measurements has been proposed using en-face OCT and OCTA. Enface OCT is used to detect the hypertransmission signal into the choroid through the area of RPE atrophy. Studies have shown that en-face imaging of GA is a reliable technique to quantify GA [33]. Moreover, the development of OCTA has allowed for GA identification, as measured by the corresponding loss of choriocapillaries. OCTA was found to display high repeatability and correlation with FAF and en-face OCT [18], which is in accordance with our results. In the same study, it was also underlined that OCTA might be superior compared to conventional FAF in detecting foveal sparing disease [18] as indicated in our study as well.

For evaluating the precision of an imaging technique in GA measurements, it is important to determine what exactly represents the area of interest. While fundus examination and fundus photography provide a gross esti- mation of the disease, as seen by indirect evidence of hypopigmented areas and "more visible" choroidal vessels [7], FAF reflects the morphological changes in the retina as low signal derived from the lack of lipofuscin in the atrophic RPE [34]. In contrast, OCTA allows for visualization of the anatomical changes due to disruption of the corresponding choriocapillaries, an option that has not been available with other conventional noninvasive technology [35]. In histological studies, GA is characterized by loss of the RPE and degeneration of the outer layers of the neurosensory retina and choriocapillaries [6]. There is still however debatable whether loss of RPE or impairment of the choriocapillaries comes first in the natural history of AMD $[36,37]$ since some authors have proposed that loss of choriocapillaries is secondary to loss of RPE in atrophic AMD [36], while others have shown by using combined light and electron microscopic approach that choriocapillary breakdown precedes degeneration of the RPE and retina in AMD [37]. More recently, it has been documented with the use of swept-source OCTA the existence of focal regions of choriocapillary flow alterations underlying areas with nascent GA, an earlier stage of GA, indicating an earlier insult to that vascular layer [38]. Additional findings to support the hypothesis that choriocapillary changes might precede RPE atrophy was recently described by another OCTA study [39].

Instead of keeping on challenging which technique is more accurate, all techniques could be embraced in an attempt to adopt multimodal imaging and to obtain indepth knowledge of the pathophysiology and prognosis of the disease. Since AMD is a progressive degenerative disease, critical variables to determine prognosis remain to be elucidated [40]. FAF patterns have been proposed to determine GA progression [35] as well as several morphologic characteristics including lesion size [41], lesion shape [42], and lesion location [43]. OCTA could also be an important tool to evaluate prognostic markers in GA patients, since it adds new visual information by administrating the retinal and the underlying choroidal circulation. It should be noted that no clear relationship between morphological changes in GA and functional outcomes has been established yet [44]. It has been proven that patches of GA correspond to absolute scotomas [45]. Therefore, it has been implicated that a foveal sparing course of GA goes along with progressive visual impairment usually associated with parafoveal scotomas [46]. Impairment may manifest as reading difficulties or difficulties in face recognition, while visual acuity is still preserved [47]. While foveal atrophy is the main determinant of BCVA in GA patients [2], the level of struc- 
tural damage on the levels of retina, RPE, and choriocapillaries, which results in central vision loss, is not well understood.

Potential limitation pertains to the limited depth penetration of OCTA in the choroid, despite the improved spatial analysis, because of the inherent signal attenuation. Therefore, imaging deeper than the choriocapillaries can sometimes be challenging, especially when acquiring a customized slab deeper than this layer in order to accurately delineate GA in these patients. In this study, we used post-processing analysis with ImageJ. This limits the application of our method in daily clinical practice for 2 reasons: first because additional time and user training are required to export images and perform calculations in third party software; second, while measurements are available with intergraded software, it is considered rather challenging to accurately delineate the atrophy borders, manually, on OCTA scans. In our study pool, none of the patients had coalescent areas of peripapillary and macular atrophy. In these cases, where measurements are typically challenging, we suggest that graders should look back to previous patient examinations to identify GA pattern, to appropriately define macular GA borders.

In conclusion, this study demonstrated the applicability of OCTA in GA evaluation in AMD patients with and without foveal sparing. We have shown that OCTA can be used for GA quantification with a simple semiautomated process. Moreover, our comparative results with IR, FAF, and en-face OCT demonstrate that OCTA might prove a promising valuable tool for GA measurement in patients with AMD as it is reliable, quick, and easy to perform. Future trials with a larger number of patients and by using more advanced OCTA devices could better explore the benefits of using OCTA in detecting and quantifying pathologies extending below the choriocapillary layer, such as GA.

\section{Statement of Ethics}

All procedures performed in studies involving human participants were in accordance with the ethical standards of the institutional and/or national research committee and with the $1964 \mathrm{Hel}-$ sinki Declaration and its later amendments or comparable ethical standards. The study was approved by the Institutional Review Board of Red Cross Hospital, Athens, Greece. All participants gave written informed consent for participation in the study. All participants gave written informed consent for their data to be published.

\section{Conflict of Interest Statement}

The authors report no conflicts of interest and report that they have no proprietary interest in any of the materials mentioned in this article. All authors certify that they have no affiliations with or involvement in any organization or entity with any financial interest (such as honoraria, educational grants, or other equity interest), or nonfinancial interest (such as personal or professional relationships, affiliations, knowledge, or beliefs) in the subject matter or materials discussed in this manuscript.

\section{Funding Sources}

The authors did not receive any funding.

\section{Author Contributions}

Stamatina Kabanarou and Irini Chatziralli conceived the study, collected data, and drafted the manuscript. George Bontzos collected data, made the statistical analysis, and drafted the manuscript. Tina Xirou, Zoi Kapsala, Eleni Dimitriou, and Panagiotis Theodossiadis collected data. All authors have read, critically revised, and approved the current version of the manuscript.

\section{Availability of Data and Material}

All data will be available upon request.

\section{References}

1 Wong WL, Su X, Li X, Cheung CM, Klein R, Cheng CY, et al. Global prevalence of age-related macular degeneration and disease burden projection for 2020 and 2040: a systematic review and meta-analysis. Lancet Glob Health. 2014;2(2):e106-16.

2 Schmitz-Valckenberg S, Nadal J, Fimmers R, Lindner M, Holz FG, Schmid M, et al. Modeling visual acuity in geographic atrophy secondary to age-related macular degeneration. Ophthalmologica. 2016;235(4):215-24.
3 Schaal KB, Rosenfeld PJ, Gregori G, Yehoshua $\mathrm{Z}$, Feuer WJ. Anatomic clinical trial endpoints for nonexudative age-related macular degeneration. Ophthalmology. 2016;123(5): 1060-79.

4 Holz FG, Sadda SR, Busbee B, Chew EY, Mitchell P, Tufail A, et al. Efficacy and safety of lampalizumab for geographic atrophy due to age-related macular degeneration: chroma and spectri phase 3 randomized clinical trials. JAMA Ophthalmol. 2018;136(6):666-77.
5 Klein ML, Ferris FL 3rd, Armstrong J, Hwang TS, Chew EY, Bressler SB, et al. Retinal precursors and the development of geographic atrophy in age-related macular degeneration. Ophthalmology. 2018;115(6):1026-31.

6 Sarks JP, Sarks SH, Killingsworth MC. Evolution of geographic atrophy of the retinal pigment epithelium. Eye. 1988;2(Pt 5):552-77. 
7 Bird AC, Bressler NM, Bressler SB, Chisholm IH, Coscas G, Davis MD, et al. An international classification and grading system for age-related maculopathy and age-related macular degeneration. The International ARM Epidemiological Study Group. Surv Ophthalmol. 1995;39(5):367-74.

8 Sunness JS, Gonzalez-Baron J, Applegate CA, Bressler NM, Tian Y, Hawkins B, et al. Enlargement of atrophy and visual acuity loss in the geographic atrophy form of age-related macular degeneration. Ophthalmology. 1999; 106(9):1768-79.

9 Garrity ST, Sarraf D, Freund KB, Sadda SR. Multimodal imaging of nonneovascular agerelated macular degeneration. Invest Ophthalmol Vis Sci. 2018;59:48-64.

$10 \mathrm{Wu}$ Z, Luu CD, Ayton LN, Goh JK, Lucci LM, Hubbard WC, et al. Optical coherence tomography-defined changes preceding the development of drusen-associated atrophy in age-related macular degeneration. Ophthalmology. 2014;121(12):2415-22.

11 Holz FG, Bellman C, Staudt S, Schütt F, Völcker HE. Fundus autofluorescence and development of geographic atrophy in agerelated macular degeneration. Invest Ophthalmol Vis Sci. 2001;42(5):1051-6.

$12 \mathrm{Hu}$ Z, Medioni GG, Hernandez M, Hariri A, Wu X, Sadda SR. Segmentation of the geographic atrophy in spectral-domain optical coherence tomography and fundus autofluorescence images. Invest Ophthalmol Vis Sci. 2013;54(13):8375-83.

13 Panthier C, Querques G, Puche N, Le Tien V, Garavito RB, Béchet $S$, et al. Evaluation of semiautomated measurement of geographic atrophy in age-related macular degeneration by fundus autofluorescence in clinical setting. Retina. 2014;34(3):576-82.

14 Schmitz-Valckenberg S, Brinkmann CK, Alten F, Herrmann P, Stratmann NK, Göbel AP, et al. Semiautomated image processing method for identification and quantification of geographic atrophy in age-related macular degeneration. Invest Ophthalmol Vis Sci. 2011;52(10):7640-6.

15 von Rückmann A, Fitzke FW, Bird AC. Distribution of fundus autofluorescence with a scanning laser ophthalmoscope. Br J Ophthalmol. 1995;79(5):407-12.

16 Wolf-Schnurrbusch UE, Wittwer VV, Ghanem R, Niederhaeuser M, Enzmann V, Framme C, et al. Blue-light versus green-light autofluorescence: lesion size of areas of geographic atrophy. Invest Ophthalmol Vis Sci. 2011;52(13):9497-502.

17 Waheed NK, Moult EM, Fujimoto JG, Rosenfeld PJ. Optical coherence tomography angiography of dry age-related macular degeneration. Dev Ophthalmol. 2016;56:91-100.

18 Corbelli E, Sacconi R, Rabiolo A, Mercuri S, Carnevali A, Querques L, et al. Optical coherence tomography angiography in the evaluation of geographic atrophy area extension. Invest Ophthalmol Vis Sci. 2017;58(12):5201-8.
19 Lindblad AS, Lloyd PC, Clemons TE, Gensler GR, Ferris FL 3rd, Klein ML, et al. Change in area of geographic atrophy in the Age-Related Eye Disease Study: AREDS report number 26. Arch Ophthalmol. 2009;127(9):1168-74.

20 Lindner M, Böker A, Mauschitz MM, Göbel AP, Fimmers R, Brinkmann CK, et al. Directional kinetics of geographic atrophy progression in age-related macular degeneration with foveal sparing. Ophthalmology. 2015;122(7): 1356-65.

21 Moult E, Choi W, Waheed NK, Adhi M, Lee $\mathrm{B}, \mathrm{Lu} \mathrm{CD}$, et al. Ultrahigh-speed swept-source OCT angiography in exudative AMD. Ophthalmic Surg Lasers Imaging Retina. 2014; 45(6):496-505.

22 Bland JM, Altman DG. Statistical methods for assessing agreement between two methods of clinical measurement. Lancet. 1986;1(8476): 307-10.

23 Heiferman MJ, Fawzi AA. Discordance between blue-light autofluorescence and nearinfrared autofluorescence in age-related macular degeneration. Retina. 2016;36:137-46.

24 Sunness JS, Margalit E, Srikumaran D, Applegate CA, Tian Y, Perry D, et al. The long-term natural history of geographic atrophy from age-related macular degeneration: enlargement of atrophy and implications for interventional clinical trials. Ophthalmology. 2007;114(2):271-7.

25 Klein R, Meuer SM, Knudtson MD, Klein BE. The epidemiology of progression of pure geographic atrophy: the Beaver Dam Eye Study. Am J Ophthalmol. 2008;146(5):692-9.

26 Sunness JS, Bressler NM, Tian Y, Alexander J, Applegate CA. Measuring geographic atrophy in advanced age-related macular degeneration. Invest Ophthalmol Vis Sci. 1999; 40(8):1761-9.

27 Scholl HP, Peto T, Dandekar S, Bunce C, Xing $\mathrm{W}$, Jenkins $\mathrm{S}$, et al. Inter- and intra-observer variability in grading lesions of age-related maculopathy and macular degeneration. Graefes Arch Clin Exp Ophthalmol. 2003; 241(1):39-47.

28 Ben Moussa N, Georges A, Capuano V, Merle B, Souied EH, Querques G. MultiColor imaging in the evaluation of geographic atrophy due to age-related macular degeneration. Br J Ophthalmol. 2015;99(6):842-7.

29 Schmitz-Valckenberg S, Jorzik J, Unnebrink K, Holz FG; FAM Study Group. Analysis of digital scanning laser ophthalmoscopy fundus autofluorescence images of geographic atrophy in advanced age-related macular degeneration. Graefes Arch Clin Exp Ophthalmol. 2002;240(2):73-8.

30 Schmitz-Valckenberg S, Fleckenstein M, Helb HM, Charbel Issa P, Scholl HP, Holz FG. In vivo imaging of foveal sparing in geographic atrophy secondary to age-related macular degeneration. Invest Ophthalmol Vis Sci. 2009; 50(8):3915-21.
31 Keilhauer CN, Delori FC. Near-infrared autofluorescence imaging of the fundus: visualization of ocular melanin. Invest Ophthalmol Vis Sci. 2006;47(8):3556-64.

32 Borrelli E, Nittala MG, Abdelfattah NS, Lei J, Hariri AH, Shi Y, et al. Comparison of shortwavelength blue-light autofluorescence and conventional blue-light autofluorescence in geographic atrophy. Br J Ophthalmol. 2019; 103(5):610-6.

33 Yehoshua Z, Rosenfeld PJ, Gregori G, Penha F. Spectral domain optical coherence tomography imaging of dry age-related macular degeneration. Ophthalmic Surg Lasers Imaging. 2010;41:6-14.

34 Delori FC, Dorey CK, Staurenghi G, Arend O, Goger DG, Weiter JJ. In vivo fluorescence of the ocular fundus exhibits retinal pigment epithelium lipofuscin characteristics. Invest Ophthalmol Vis Sci. 1995;36(3):718-29.

35 Holz FG, Bindewald-Wittich A, Fleckenstein M, Dreyhaupt J, Scholl HP, Schmitz-Valckenberg S, et al. Progression of geographic atrophy and impact of fundus autofluorescence patterns in age-related macular degeneration. Am J Ophthalmol. 2007;143(3):463-72.

36 Bhutto I, Lutty G. Understanding age-related macular degeneration (AMD): relationships between the photoreceptor/retinal pigment epithelium/Bruch's membrane/choriocapillaris complex. Mol Aspects Med. 2012;33(4): 295-317.

37 Biesemeier A, Taubitz T, Julien S, Yoeruek E, Schraermeyer U. Choriocapillaris breakdown precedes retinal degeneration in age-related macular degeneration. Neurobiol Aging. 2014;35(11):2562-73.

38 Moult EM, Waheed NK, Novais EA, Choi W, Lee B, Ploner SB, et al. Swept-source optical coherence tomography angiography reveals choriocapillaris alterations in eyes with nascent geographic atrophy and drusen-associated geographic atrophy. Retina. 2016; 36(Suppl 1):S2-11.

39 Sacconi R, Corbelli E, Carnevali A, Querques L, Bandello F, Querques G. Optical coherence tomography angiography in geographic atrophy. Retina. 2018;38(12):2350-5.

40 Pfau M, Lindner M, Goerdt L, Thiele S, Nadal J, Schmid M, et al. Prognostic value of shapedescriptive factors for the progression of geographic atrophy secondary to age-related macular degeneration. Retina. 2019;39(8): 1527-40.

41 Joachim N, Mitchell P, Kifley A, Rochtchina $\mathrm{E}$, Hong $\mathrm{T}$, Wang JJ. Incidence and progression of geographic atrophy: observations from a population-based cohort. Ophthalmology. 2013;120(10):2042-50.

42 Biarnés M, Arias L, Alonso J, Garcia M, Hijano $\mathrm{M}$, Rodríguez A, et al. Increased fundus autofluorescence and progression of geographic atrophy secondary to age-related macular degeneration: the GAIN study. Am J Ophthalmol. 2015;160(2):345-e5. 
43 Mauschitz MM, Fonseca S, Chang P, Göbel AP, Fleckenstein M, Jaffe GJ, et al. Topography of geographic atrophy in age-related macular degeneration. Invest Ophthalmol Vis Sci. 2012;53(8):4932-9.

44 Holz FG, Strauss EC, Schmitz-Valckenberg S, van Lookeren Campagne M. Geographic atrophy: clinical features and potential therapeutic approaches. Ophthalmology. 2014; 121(5):1079-91.
45 Sunness JS, Schuchard RA, Shen N, Rubin GS, Dagnelie G, Haselwood DM. Landmark-driven fundus perimetry using the scanning laser ophthalmoscope. Invest Ophthalmol Vis Sci. 1995;36(9):1863-74.

46 Forte R, Querques G, Querques L, Leveziel N, Benhamou N, Souied EH. Multimodal evaluation of foveal sparing in patients with geographicatrophy due to age-related macular degeneration. Retina. 2013;33(3):482-9.
47 Sunness JS, Rubin GS, Applegate CA, Bressler NM, Marsh MJ, Hawkins BS, et al. Visual function abnormalities and prognosis in eyes with age-related geographic atrophy of the macula and good visual acuity. Ophthalmology. 1997;104(10):1677-91. 